\title{
Number of Digits in Two Integers and Their Multiplication
}

\author{
Xingbo WANG \\ Department of Mechatronic Engineering, Foshan University, Foshan, China \\ Guangdong Engineering Center of Information Security for Intelligent Manufacturing System, Foshan, China \\ State Key Laboratory of Mathematical Engineering and Advanced Computing, Wuxi, China \\ Email: dr.xbang@qq.com; xbwang@fosu.edu.cn
}

\begin{abstract}
This paper first proves a formula that discloses the relationship between the number of digits in multiplication and its two divisors, then proves that the two divisors are of the equal length if the divisor-ratio generated from the bigger divisor divided by the small one is smaller than 10 . Hence the paper shows that, all the RSA numbers must have two divisors of the equal length.
\end{abstract}

Keywords: Number of digits, multiplication, divisor, RSA number.

\section{Introduction}

Analysing the arithmetic calculations, $1035918371=32717 \times 31663,170442776634553=228479 \times 745988807$ and $1123877887715932507=299155897 \times 3756830131$, one can see that, the semiprimes, 1035918371 , 170442776634553 and 1123877887715932507 are decimal integer with 10, 15 and 19 decimal digits respectively, and the number of the digits in the 6 divisors of the 3 semiprimes are $(5,5),(6,9)$ and $(9,10)$ respectively. Coincidentally, $10=5+5,15=6+9$ and $19=9+10$. Are these really coincidental? Look at more examples list in Table 1.

Table 1. Number of digits in semiprimes and their divisors

\begin{tabular}{lc}
\hline \multicolumn{1}{c}{$\mathrm{m}=\mathrm{p} \times \mathrm{q}$} & $\operatorname{digit}(\mathrm{m}), \operatorname{digit}(\mathrm{p}), \operatorname{digit}(\mathrm{q})$ \\
\hline $16637=131 \times 127$ & $5,3,3$ \\
$2129189=2003 \times 1063$ & $7,4,4$ \\
$4538873=2237 \times 2029$ & $7,4,4$ \\
$8772041=3299 \times 2659$ & $7,4,4$ \\
$1035918371=32717 \times 31663$ & $10,5,5$ \\
$2512642129=51071 \times 49199$ & $10,5,5$ \\
$5783560579=81017 \times 71387$ & $10,5,5$ \\
$9048212729=99871 \times 90599$ & $10,5,5$ \\
$80735174503=311393 \times 259271$ & $11,6,6$ \\
$211041144109=511279 \times 412771$ & $12,6,6$ \\
$170442776634553=228479 \times 745988807$ & $15,6,9$ \\
$1808898276844231=2424833 \times 745988807$ & $16,7,9$ \\
$35249679931198483=59138501 \times 596052983$ & $17,8,9$ \\
$37522676526028537=193707721 \times 193707697$ & $17,9,9$ \\
$556499304645216091=745988813 \times 745988807$ & $18,9,9$ \\
$1123877887715932507=299155897 \times 3756830131$ & $19,9,10$ \\
$1129367102454866881=25869889 \times 43655660929$ & $19,8,11$ \\
$1902408569846737793=745988807 \times 2550183799$ & $19,9,10$ \\
$10188337563435517819=70901851 \times 143696355169$ & $20,8,12$ \\
$24928816998094684879=347912923 \times 71652460573$ & $20,9,11$ \\
\hline
\end{tabular}

Apart from the examples in Table 1, analyzing the RSA numbers [1] yields the following Table 2. 
Table 2. Number of digits in RSA numbers and their divisors

\begin{tabular}{ccccc}
\hline No. & RSA numbers & D $_{\text {RSA }}$ & $D_{\mathrm{p}}$ & $\mathrm{D}_{\mathrm{q}}$ \\
\hline 1 & RSA100 & 100 & 50 & 50 \\
2 & RSA110 & 110 & 55 & 55 \\
3 & RSA120 & 120 & 60 & 60 \\
4 & RSA129 & 129 & 64 & 66 \\
5 & RSA130 & 130 & 65 & 65 \\
6 & RSA140 & 140 & 70 & 70 \\
7 & RSA150 & 150 & 75 & 75 \\
8 & RSA155 & 155 & 78 & 78 \\
9 & RSA160 & 160 & 80 & 80 \\
10 & RSA170 & 170 & 85 & 85 \\
11 & RSA180 & 180 & 90 & 90 \\
12 & RSA190 & 190 & 95 & 95 \\
13 & RSA200 & 200 & 100 & 100 \\
14 & RSA210 & 210 & 105 & 105 \\
15 & RSA220 & 220 & 110 & 110 \\
16 & RSA576 & 174 & 87 & 87 \\
17 & RSA640 & 193 & 97 & 97 \\
18 & RSA704 & 212 & 106 & 106 \\
19 & RSA768 & 232 & 116 & 116 \\
20 & RSA230 & 230 & 115 & 115 \\
\hline
\end{tabular}

Obviously, it seems to have the following formula

$$
D_{m}=D_{p}+D_{q} \text { or } D_{m}=D_{p}+D_{q}-1
$$

where $D_{p}, D_{q}$ and $D_{m}$ mean number of digits in divisor $p$, divisor $q$ and their multiplication $m=p \times q$ respectively.

Now comes a question, is the formula (1) true? And under what condition it is true if it is true? This paper answers the questions.

\section{$2 \quad$ Preliminaries}

The floor function of real number $x$ is denoted by symbol $\lfloor x\rfloor$ that satisfies $\lfloor x\rfloor \leq x<\lfloor x\rfloor+1$; the fraction part of $x$ is denoted by symbol $\{x\}$ that satisfies $x=\lfloor x\rfloor+\{x\}$. Symbol $D_{n}$ is the decimal digits of positive integer $n$. In this whole article, $A \Rightarrow B$ means conclusion $B$ can be derived from condition $A ; A \Leftrightarrow B$ means $B$ holds if and only if $A$ holds. Symbol $A \oplus B$ means $A$ or $B$ holds.

Lemma 1. (See in [2]) A positive integer $n$ with base $b$ has $\left\lfloor\log _{b} n\right\rfloor+1$ digits.

Lemma 2. (See in [3]) Let $x$ and $y$ be real numbers; then

$$
\begin{aligned}
& \text { (P1) }\lfloor x\rfloor+\lfloor y\rfloor \leq\lfloor x+y\rfloor \leq\lfloor x\rfloor+\lfloor y\rfloor+1 \\
& \text { (P2) }\lfloor x\rfloor-\lfloor y\rfloor-1 \leq\lfloor x-y\rfloor \leq\lfloor x\rfloor-\lfloor y\rfloor<\lfloor x\rfloor-\lfloor y\rfloor+1 \\
& \text { (P3) }\lfloor 2 x\rfloor+\lfloor 2 y\rfloor \geq\lfloor x\rfloor+\lfloor y\rfloor+\lfloor x+y\rfloor \\
& \text { (P13) } x \leq y \Rightarrow\lfloor x\rfloor \leq\lfloor y\rfloor \\
& \text { (P20) }\lfloor\sqrt{x}\rfloor=\lfloor\sqrt{\lfloor x\rfloor}\rfloor \text { with } x \geq 0 \\
& \text { (P21) }\left\lfloor\log _{b} x\right\rfloor=\left\lfloor\log _{b}\lfloor x\rfloor\right\rfloor \text { with } x>0
\end{aligned}
$$

Lemma 3. (See in [4]) Let $\alpha$ and $x$ be positive real numbers; then it holds

$$
\alpha\lfloor x\rfloor-1<\lfloor\alpha x\rfloor<\alpha(\lfloor x\rfloor+1)
$$

Particularly, if $\alpha$ is a positive integer, say $\alpha=n$, then it yields 


$$
n\lfloor x\rfloor \leq\lfloor n x\rfloor \leq n(\lfloor x\rfloor+1)-1
$$

\section{$3 \quad$ Main Results and Proofs}

Theorem 1. Let $m, p$ and $q$ be positive integers and $m=p q$; then

$$
D_{p}+D_{q}-1 \leq D_{m} \leq D_{p}+D_{q}
$$

Proof. It yields by the given conditions and Lemma 2

$$
m=p q \Rightarrow D_{m}=\left\lfloor\log _{10} p q\right\rfloor+1=\left\lfloor\log _{10} p+\log _{10} q\right\rfloor+1 \leq\left\lfloor\log _{10} p\right\rfloor+\left\lfloor\log _{10} q\right\rfloor+2=D_{p}+D_{q}
$$

Meanwhile, since $\left\lfloor\log _{10} p\right\rfloor+\left\lfloor\log _{10} q\right\rfloor \leq\left\lfloor\log _{10} p+\log _{10} q\right\rfloor$, it holds

Thereby it holds

$$
D_{m}=\left\lfloor\log _{10} p q\right\rfloor+1=\left\lfloor\log _{10} p+\log _{10} q\right\rfloor+1 \geq\left\lfloor\log _{10} p\right\rfloor+\left\lfloor\log _{10} q\right\rfloor+1=D_{p}+D_{q}-1
$$

which is just the (2).

$$
D_{p}+D_{q}-1 \leq D_{m} \leq D_{p}+D_{q}
$$

Theorem 2. Suppose $m$ is a positive integer and $s=\lfloor\sqrt{m}\rfloor$; then

$$
2 D_{s}-1 \leq D_{m} \leq 2 D_{s}
$$

Proof. By the given conditions and Lemma 2 (P21) as well as Lemma 3, it leads to

$$
m=\sqrt{m} \cdot \sqrt{m} \Rightarrow D_{m}=\left\lfloor\log _{10}(\sqrt{m} \cdot \sqrt{m})\right\rfloor+1=\left\lfloor 2 \log _{10} \sqrt{m}\right\rfloor+1=\left\lfloor 2 \log _{10}\lfloor\sqrt{m}\rfloor\right\rfloor+1=\left\lfloor 2 \log _{10} s\right\rfloor+1
$$

That is

which is

$$
2\left\lfloor\log _{10} s\right\rfloor+1 \leq D_{m} \leq 2\left(\left\lfloor\log _{10} s\right\rfloor+1\right)-1+1=2\left(\left\lfloor\log _{10} s\right\rfloor+1\right)
$$

$$
2 D_{s}-1 \leq D_{m} \leq 2 D_{s}
$$

Example 1. Choose 13 integers by

$$
\text { Od }:=\operatorname{Array}([16637,2129189,4538873,8772041,
$$

1035918371, 2512642129, 5783560579, 9048212729, 80735174503,

211041144109, 170442776634553, 1808898276844231, 35249679931198483])

Program in Maple with the following codes

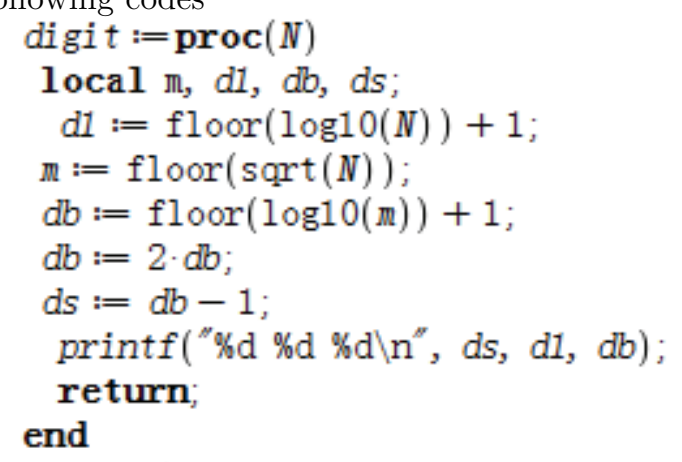

And run the program with command

for $k$ to 13 do digit $(O d(k))$ end do

The output is as follows 


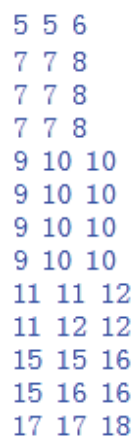

The output matches to the Theorem 2 .

Corollary 1. Suppose $m$ is a positive integers and $s=\lfloor\sqrt{m}\rfloor$; then

$$
D_{s}=\left\{\begin{array}{l}
\frac{D_{m}}{2}, D_{m} \text { is even } \\
\frac{D_{m}+1}{2}, D_{m} \text { is odd }
\end{array}\right.
$$

Proof. Since $D_{m}$ is an integer, the inequality (3) shows that, when $D_{m}$ is even it is equal to $2 D_{s}$ while it is equal to $2 D_{s}-1$ when it is odd.

Theorem 2. Suppose $m, p$ and $q$ are positive integers with $1<p \leq q$; let $m=p q$ and $k=\frac{q}{p}$; then

$$
D_{q}=\left\{\begin{array}{c}
\frac{D_{m}}{2} \oplus\left(\frac{D_{m}}{2}+1\right), D_{m} \text { is even } \\
\frac{D_{m}+1}{2}, D_{m} \text { is odd }
\end{array}\right.
$$

Proof. By the given conditions it yields

$$
m=p q \Rightarrow D_{m}=D_{p q}=\left\lfloor\log _{10}(p q)\right\rfloor+1=\left\lfloor\log _{10}\left(\frac{q^{2}}{k}\right)\right\rfloor+1=\left\lfloor 2 \log _{10} q-\log _{10} k\right\rfloor+1
$$

By Lemma 1 (P2), it yields

$$
\left\lfloor 2 \log _{10} q\right\rfloor-\left\lfloor\log _{10} k\right\rfloor \leq D_{m} \leq\left\lfloor 2 \log _{10} q\right\rfloor-\left\lfloor\log _{10} k\right\rfloor+1
$$

Note that, $k<10$ yields $\left\lfloor\log _{10} k\right\rfloor=0$; hence it holds

By Lemma 3, it holds

$$
\left\lfloor 2 \log _{10} q\right\rfloor \leq D_{m} \leq\left\lfloor 2 \log _{10} q\right\rfloor+1
$$

That is

$$
2\left\lfloor\log _{10} q\right\rfloor \leq D_{m} \leq 2\left(\left\lfloor\log _{10} q\right\rfloor+1\right)
$$

$$
2\left(D_{q}-1\right) \leq D_{m} \leq 2 D_{q}
$$

Since $D_{m}$ is an integer, it takes $2 D_{q}-1$ when it is odd. Thereby, when $D_{m}$ is odd, $D_{q}=\frac{D_{m}+1}{2}$, whereas, when it is even $D_{q}=\frac{D_{m}}{2} \oplus\left(\frac{D_{m}}{2}+1\right)$

Theorem 3. Suppose $m, p$ and $q$ are positive integers with $1<p \leq q$; let $m=p q$ and $k=\frac{q}{p}$; then $1 \leq k<10$ yields $D_{p}=D_{q}$.

Proof. From the given conditions, it can see 


$$
m=p q \Rightarrow m=k p^{2} \Rightarrow\left\{\begin{array}{l}
p^{2}=\frac{m}{k} \Rightarrow 2 \log _{10} p=\log _{10} m-\log _{10} k \\
q^{2}=k m \Rightarrow 2 \log _{10} q=\log _{10} m+\log _{10} k
\end{array}\right.
$$

Since $k<10$ yields $\left\lfloor\log _{10} k\right\rfloor=0$; hence when $k<10$ it holds

$$
\log _{10} p=\log _{10} q
$$

which naturally results in

$$
D_{p}=D_{q}
$$

Corollary 2. Suppose $m, p$ and $q$ are positive integers with $1<p \leq q$; let $m=p q$ and $k=\frac{q}{p}$; then $1 \leq k<10$ yields

$$
D_{p}=D_{q}=\left\{\begin{array}{c}
\frac{D_{m}}{2} \oplus\left(\frac{D_{m}}{2}+1\right), D_{m} \text { is even } \\
\frac{D_{m}+1}{2}, D_{m} \text { is odd }
\end{array}\right.
$$

Proof. (Omitted).

Example 2. Look at Table 1 and analyze the data in the table, it can see that, all the data fit Corollary 2. In addition, take the RSA numbers as examples, analyze their divisor-ratios and number of

\begin{tabular}{|c|c|c|c|c|c|}
\hline No. & RSA numbers & $\mathrm{k}=\mathrm{q} / \mathrm{p}$ & $\mathrm{D}_{\mathrm{RSA}}$ & $\mathrm{D}_{\mathrm{p}}$ & $\mathrm{D}_{\mathrm{q}}$ \\
\hline 1 & RSA100 & 1.056 & 100(even) & 50 & 50 \\
\hline 2 & RSA110 & 1.047 & 110 (even) & 55 & 55 \\
\hline 3 & RSA120 & 2.118 & 120(even) & 60 & 60 \\
\hline 4 & RSA129 & $93.880>10$ & 129 (odd) & 64 & 66 \\
\hline 5 & RSA130 & 1.147 & 130 (even) & 65 & 65 \\
\hline 6 & RSA140 & 1.843 & 140 (even) & 70 & 70 \\
\hline 7 & RSA150 & 1.281 & 150(even) & 75 & 75 \\
\hline 8 & RSA155 & 1.039 & 155(even) & 78 & 78 \\
\hline 9 & RSA160 & 1.043 & 160 (even) & 80 & 80 \\
\hline 10 & RSA170 & 1.188 & 170 (even) & 85 & 85 \\
\hline 11 & RSA180 & 1.190 & 180(even) & 90 & 90 \\
\hline 12 & RSA190 & 1.897 & 190(even) & 95 & 95 \\
\hline 13 & RSA200 & 2.244 & 200(even) & 100 & 100 \\
\hline 14 & RSA210 & 1.290 & 210 (even) & 105 & 105 \\
\hline 15 & RSA220 & 2.084 & 220 (even) & 110 & 110 \\
\hline 16 & RSA576 & 1.188 & 174(even) & 87 & 87 \\
\hline 17 & RSA640 & 1.163 & 193(odd) & 97 & 97 \\
\hline 18 & RSA704 & 1.116 & 212 (even) & 106 & 106 \\
\hline 19 & RSA768 & 1.098 & 232 (even) & 116 & 116 \\
\hline 20 & RSA230 & 1.141 & 230(even) & 115 & 115 \\
\hline
\end{tabular}
digits in the RSA numbers and their divisors; the results are list in Table 3. It can see these data completely fit Corollary 2.

Table 3. RSA numbers, their divisor-ratios plus related number of digits

Corollary 3. All the RSA numbers must have two divisors of the equal length.

Proof. By the American Digital Signature Standard (DSS)[5], an RSA number, is a big semiprime composed of two distinct prime divisors, say $p$ and $q$ with $3 \leq p<q$ such that $1<q / p<\sqrt{2}$. By Corollary 2 , it is sure that all the RSA numbers must have two divisors of the equal length. 


\section{Conclusion}

Knowing the relationship between multiplication and its factors helps know the range of the factors in practice of integer factorization. By the conclusions drawn in this paper, it is known that the two divisors of an RSA number are of the equal length. This provides a mathematical foundation in knowing of the RSA numbers. Hope to be a benefit to the researchers.

Acknowledgments. The research work is supported by the State Key Laboratory of Mathematical Engineering and Advanced Computing under Open Project Program No.2017A01, Department of Guangdong Science and Technology under project 2015A010104011, Foshan Bureau of Science and Technology under projects 2016AG100311, Project gg040981 from Foshan University. The authors sincerely present thanks to them all.

\section{References}

1. wikipedia. RSA number, https://en.wikipedia.org/wiki/RSA_numbers

2. K H Rosen. Elementary Number Theory 83 Its Application (6th eds), Addison-Wesley ,2010, pp.66

3. X WANG. "Brief Summary of Frequently-Used Properties of the Floor Function," IOSR Journal of Mathematics, vol. 13, no. 5, pp. 46 - 48, 2017.

4. X WANG, "Some New Inequalities With Proofs and Comments on Applications," Journal of Mathematics Research, vol. 11, no. 3, pp. 15-19, 2018

5. National Institute of Standards and Technology (NIST). Digital signature standard (DSS), FIPS publication 186-3, June 2009 . 